\title{
Breast cancer is associated to impaired glucose/insulin homeostasis in premenopausal obese/overweight patients
}

\author{
Raúl M. Luque ${ }^{1,2,3,4,5, *, \#}$, Laura M. López-Sánchez ${ }^{1,2,3,4,5, *}$, Alicia Villa-Osaba $a^{1,2,3,4,5, *}$, \\ Isabel M. Luque ${ }^{1,3}$, Ana L. Santos-Romero ${ }^{1,4,6}$, Elena M. Yubero-Serrano ${ }^{1,4,7}$, María \\ Cara-García ${ }^{1,4,6}$, Marina Álvarez-Benito ${ }^{1,4,6}$, José López-Miranda ${ }^{1,4,7}$, Manuel D. \\ Gahete $^{1,2,3,4,5, \#}$ and Justo P. Castaño $\mathbf{1}^{1,2,3,4,5, \#}$ \\ ${ }^{1}$ Maimonides Institute of Biomedical Research of Cordoba (IMIBIC), Córdoba, Spain \\ ${ }^{2}$ Department of Cell Biology, Physiology and Immunology, University of Córdoba, Córdoba, Spain \\ ${ }^{3}$ Hospital Universitario Reina Sofia, Córdoba, Spain \\ ${ }^{4}$ CIBERobn, Córdoba, Spain \\ ${ }^{5}$ ceiA3, Córdoba, Spain \\ ${ }^{6}$ Mammary Gland Unit, Hospital Universitario Reina Sofía, Córdoba, Spain \\ ${ }^{7}$ Lipids and Atherosclerosis Unit, Hospital Universitario Reina Sofía, Córdoba, Spain \\ *These authors contributed equally to this work \\ \#These authors co-directed the work and should be considered co-senior authors \\ Correspondence to: Raúl M. Luque, email: raul.luque@uco.es \\ Manuel D. Gahete, email: bc2gaorm@uco.es \\ Justo P. Castaño, email: justo@uco.es
}

Keywords: breast cancer, glucose, insulin, OGTT, obesity

Received: June 27, $2017 \quad$ Accepted: July 25, 2017

Published: August 23, 2017

Copyright: Luque et al. This is an open-access article distributed under the terms of the Creative Commons Attribution License 3.0 (CC BY

3.0), which permits unrestricted use, distribution, and reproduction in any medium, provided the original author and source are credited.

\section{ABSTRACT}

The association between breast cancer (BCa) presence and altered glucose/ insulin metabolism is controversial likely due to an inaccurate insulin resistance (IR) assessment and inappropriate stratification of patients by body-mass index (BMI) and menopausal state. 148 women with suspect of sporadic BCa were stratified by BMI and menopause. Fasting levels of glucose, insulin, glycohemoglobin and selected IR-related and tumor-derived markers were measured. Glucose/insulin levels during OGTT were used to calculate insulin resistance/sensitivity indexes. Analysis of 77 BCa-bearing patients and 71 controls showed an association between BCa and IR as demonstrated by impaired glucose/insulin homeostasis (increased fasting- and OGTT-induced glucose levels) and deteriorated IR indexes, which was especially patent in premenopausal women. The association between BCa presence and IR was markedly influenced by BMI, being obese BCa patients significantly more insulin resistant than controls. BCa presence was associated to elevated levels of IR (glucose, triglycerides) and tumor-derived (VEGF) markers, especially in overweight/obese patients. BCa presence is associated to IR in overweight/obese premenopausal but not in premenopausal normal weight or postmenopausal women. Our data support a bidirectional relationship between dysregulated/imbalanced glucose/insulin metabolism and $\mathrm{BCa}$, as tumor- and IR-markers are correlated with the impairment of glucose/insulin metabolism in overweight/obese premenopausal BCa patients. 


\section{INTRODUCTION}

Breast cancer $(\mathrm{BCa})$ is the most frequent and the second leading cause of cancer-related death in female population [1]. $\mathrm{BCa}$ is conditioned by genetic predisposition and environmental factors, such as obesity [2-5], diabetes [6], or metabolic syndrome [6-8]. All these $\mathrm{BCa}$-associated risk/prognostic factors share several hallmarks, wherein insulin resistance (IR) seems to have particular relevance $[9,10]$. Indeed, IR is associated to hyperinsulinemia, hypertension, and impaired glucose tolerance, and, consequently, has been proposed as a putative underlying cause in the relationship of obesity, diabetes, and metabolic syndrome with higher $\mathrm{BCa}$ risk $[2,8,11,12]$, prognosis $[7,13]$ and survival $[3,14]$. However, the direct, causative association between IR and $\mathrm{BCa}$ is still controversial [15-19] in that, although fasting glucose seems to be a risk factor for $\mathrm{BCa}[20]$, data regarding other IR markers (insulin/C-peptide) and their role in $\mathrm{BCa}$ development/progression are inconclusive, as some studies reported an association $[16,18]$, whereas others did not $[15,17,19]$. It is also controversial whether $\mathrm{BCa}$ presence is associated to IR and/or dysregulated or imbalanced glucose and insulin metabolism. Indeed, although a recent study reported higher fasting glucose and insulin levels in non-diabetic $\mathrm{BCa}$ patients [13], a previous meta-analysis found no alteration of IR markers in $\mathrm{BCa}$ patients [21]. In this scenario, it has been suggested that the lack of convincing data regarding a putative association between IR and $\mathrm{BCa}$ could be due to the fact that IR has not been appropriately evaluated and/or analyzed. Thus, some authors have proposed that oral glucose tolerance test (OGTT) should be used, for impairments in glucose metabolism are better identified dynamically, in response to a glucose challenge $[21,22]$. Additionally, studies analyzing the relationship between IR and $\mathrm{BCa}$ could be biased by a lack of correct patient stratification in terms of body-mass index (BMI) and menopausal state, in that both factors are directly associated to IR and BCa [23-25]. Finally, it should be noted that several other IR-related factors are associated to $\mathrm{BCa}$ [altered lipids, cytokines, adipokines, insulinlike growth factor I (IGF-I), Tumor necrosis factor alpha (TNF-alpha), pro-angiogenic factors, estrogens, etc.], which could be modulating/masking the putative direct relationship between IR and $\mathrm{BCa}$ [12].

Accordingly, in an attempt to unequivocally elucidate the association between $\mathrm{BCa}$ and glucose/ insulin metabolism, by more accurately assessing IR, and by appropriately stratifying the patients by BMI and menopausal state, we have implemented a prospective study including 148 women with suspect of non-familial, sporadic $\mathrm{BCa}$ in whom we measured glucose, insulin, glycohemoglobin, and other IR-related and tumor-derived markers, as well as glucose/insulin levels during the OGTT. Our initial hypothesis was that there is a marked association between $\mathrm{BCa}$ presence and alterations in glucose/insulin metabolism, which could be influenced by BMI and menopausal status.

\section{RESULTS}

\section{Study population}

A total of 148 women with suspect of non-familial, sporadic $\mathrm{BCa}$ were included in this prospective study (77 $\mathrm{BCa}$ patients and 71 controls). Demographic and clinical data are shown in Table 1. Mean age $(p<0.001)$ and postmenopausal status proportion $(p=0.001)$ were higher in $\mathrm{BCa}$ group compared to controls, whereas obesityrelated parameters (body weight, BMI, waist circumference or cholesterol levels) did not differ (Table 1).

\section{BCa patients exhibited impaired glucose/insulin metabolism}

BCa patients presented significantly higher fasting levels of glucose and glycohemoglobin compared to controls, without changes in fasting insulin (Figure 1A). Additionally, OGTT was drastically impaired in BCa patients (BCa presence effect: $p=0.014$ ) in that glucose levels remained more elevated in $\mathrm{BCa}$ patients at 60,90 and $120 \mathrm{~min}$ after glucose load, with no changes during the first phase (0-30 min), suggesting peripheral IR (Figure 1B). Similarly, insulin levels remained more elevated in BCa patients during OGTT at 90 and 120 min (Figure 1B). Accordingly, Simple Matsuda Index (SMI) was lower in BCa individuals; while the Homeostatic Model Assessment of Insulin Resistance (HOMA-IR) and the Hepatic Insulin Resistance Index (HIRI) of BCa patients were numerically but not significantly elevated $(p=0.392$ and $p=0.472$, respectively) compared to controls (Figure 1C). Remarkably, tumor type or receptor status had no significant effect on these associations (data not shown).

Subsequently, as menopause could be associated with $\mathrm{BCa}$, obesity, and glucose/insulin metabolism, we subdivided both groups by menopausal state (Supplementary Table 1). This analysis revealed that premenopausal $\mathrm{BCa}$ patients had higher glucose and glycohemoglobin levels and impaired OGTTs compared to premenopausal controls (Figure 2); oppositely, no differences were found between postmenopausal patients. In addition, premenopausal $\mathrm{BCa}$ patients presented a nonsignificant trend for higher HIRI ( $p=0.08$; Figure 2). Of note, postmenopausal controls exhibited increased glucose and glycohemoglobin levels, and impaired OGTTs compared to premenopausal controls (Supplementary Figure 1).

As age, a risk factor for $\mathrm{BCa}$ and obesity incidence, was higher in BCa patients (Table 1 and Supplementary Table 1), general linear models for repeated measurements were implemented to clarify its putative influence on the 
Table 1: Demographic and clinical parameters of the cohort

\begin{tabular}{lccc}
\hline & $\begin{array}{c}\text { Control } \\
(\boldsymbol{n}=\mathbf{7 1})\end{array}$ & $\begin{array}{c}\text { Breast cancer } \\
(\boldsymbol{n}=\mathbf{7 7})\end{array}$ & $\boldsymbol{p}$-value \\
\hline Age (years) & $44.0 \pm 1.3$ & $53.6 \pm 1.2$ & $<\mathbf{0 . 0 0 1}$ \\
Weight $(\mathrm{kg})$ & $67.9 \pm 1.3$ & $69.2 \pm 1.2$ & 0.480 \\
BMI $\left(\mathrm{kg} / \mathrm{m}^{2}\right)$ & $26.2 \pm 0.5$ & $26.9 \pm 0.5$ & 0.316 \\
Systolic pressure (mm Hg) & $112.7 \pm 1.9$ & $127.0 \pm 2.6$ & $<\mathbf{0 . 0 0 1}$ \\
Diastolic pressure (mm Hg) & $69.7 \pm 1.4$ & $75.4 \pm 1.8$ & $\mathbf{0 . 0 3 4}$ \\
Waist perimeter (cm) & $86.3 \pm 1.3$ & $89.1 \pm 1.4$ & 0.160 \\
Number of children & $1.9 \pm 0.09$ & $2.3 \pm 0.1$ & 0.124 \\
Age of menarche & $12.3 \pm 0.2$ & $12.6 \pm 0.1$ & 0.241 \\
Age of first childbirth & $26.2 \pm 0.7$ & $25.0 \pm 0.6$ & 0.221 \\
Premenopausal state (\%) & 77.0 & 55.0 & $\mathbf{0 . 0 0 1}$ \\
Postmenopausal state (\%) & 23.0 & 45.0 & $\mathbf{0 . 0 0 1}$ \\
Total cholesterol (mg/dL) & $201.2 \pm 4.4$ & $210.6 \pm 3.9$ & 0.116 \\
HDL cholesterol (mg/dL) & $53.6 \pm 1.5$ & $53.8 \pm 1.4$ & 0.950 \\
LDL cholesterol (mg/dL) & $127.5 \pm 3.7$ & $134.6 \pm 3.6$ & 0.109 \\
\hline Values & & & \\
\hline
\end{tabular}

Values represent mean \pm SEM of each parameter in control $(n=71)$ and breast cancer $(n=77)$ groups. $P$-value indicates differences by Mann-Whitney, Student $t$-test and/or Fisher exact test. $p<0.05$ was considered significant.

parameters evaluated. Considering the whole cohort, this analysis confirmed a significant effect of BCa presence on OGTT response. However, this effect disappeared when age was used as covariate, indicating an effect of age on OGTT response (Supplementary Table 2). Furthermore, this model confirmed a significant effect of BCa presence in premenopausal women, which was maintained when corrected by age (Supplementary Table 2). This data was further validated by selecting a subgroup of premenopausal age-matched control and breast cancer patients (average age of 44.0 and 44.4 years, respectively; $p$-value $=0.709$ by Student $t$-test) and analyzing OGTT response, which confirmed a significant effect of $\mathrm{BCa}$ on OGTT response in premenopausal women when corrected by age (Supplementary Figure 2).

\section{Glucose/insulin metabolism was altered in obese, but not in normal weight, BCa patients}

When normal weight $\left(\right.$ BMI $<25 \mathrm{~kg} / \mathrm{m}^{2}$ ) and overweight/obese $\left(B M I \geq 25 \mathrm{~kg} / \mathrm{m}^{2}\right)$ patients were considered separately, a strong association between $\mathrm{BCa}$ and impaired glucose/insulin homeostasis was observed in overweight/obese individuals but not in normal weight women (Figure 3). Fasting glucose and insulin levels were affected by body weight, but only fasting glucose levels were influenced by $\mathrm{BCa}$ presence, being significantly more elevated in overweight/obese (BMI $\geq 25) \mathrm{BCa}$ patients than in normal weight $\mathrm{BCa}$ patients, and, importantly, than in overweight/obese (BMI $\geq 25$ ) controls (Figure $3 \mathrm{~A}$ ). Furthermore, whereas OGTT was not altered in BCabearing normal weight patients compared to normal weight controls (Figure 3B), BCa patients with $\mathrm{BMI} \geq 25$ showed impaired OGTT compared to obese controls, as glucose levels remained significantly elevated at 30,60,90 and $120 \mathrm{~min}$, and insulin levels did not recover after $60 \mathrm{~min}$, reaching a significant difference at $120 \mathrm{~min}$ vs. obese controls (Figure 3B). Indeed, IR indexes were clearly influenced by body weight within both, control and $\mathrm{BCa}$ groups (Figure 3C); however, despite the overt numerical differences, only non-significant trends were found between overweight/obese $\mathrm{BCa}$ patients and controls (Figure 3C).

Remarkably, premenopausal $\mathrm{BCa}$ patients had a more pronounced impairment of glucose/insulin metabolism than their controls, especially in patients with BMI $\geq 25$ (Supplementary Figure 3). In fact, fasting glucose and glycohemoglobin levels were not altered in $\mathrm{BCa}$ normal weight premenopausal women compared to controls, but tended to be higher (glucose; $p=0.072$ ) or were significantly elevated (glycohemoglobin; $p<0.05$ ) in $\mathrm{BCa}$ premenopausal patients with $\mathrm{BMI}>25$ compared to controls (Supplementary Figure 3A). In contrast, fasting glucose was significantly lower in $\mathrm{BCa}$ normal weight postmenopausal patients compared to controls (Supplementary Figure 3A). Accordingly, overall glucose levels were not significantly altered during the OGTT by $\mathrm{BCa}$ presence in premenopausal normal weight subjects $(p=0.153)$, despite slightly elevated glucose levels at 60 and 90 min in BCa patients vs. controls (Supplementary Figure 3B). Conversely, $\mathrm{BCa}$ presence was clearly associated to impaired OGTT in premenopausal $\mathrm{BCa}$ patients with $\mathrm{BMI} \geq 25$ compared to premenopausal controls, with higher glucose levels at 30, 60, 90 and 120 min (Supplementary Figure 3B). In contrast, no body 
weight-related differences were found for glucose in OGTT between BCa vs. control in postmenopausal women (Supplementary Figure 3B). Similarly, only premenopausal $\mathrm{BCa}$ patients with $\mathrm{BMI} \geq 25$ showed a non-significant trend for elevated insulin levels after $120 \min (p=0.083)$ (Supplementary Figure 3B). Furthermore, SMI tended to be reduced in $\mathrm{BCa}$ compared to controls in premenopausal women ( $p=0.053$, two-way ANOVA), but not in
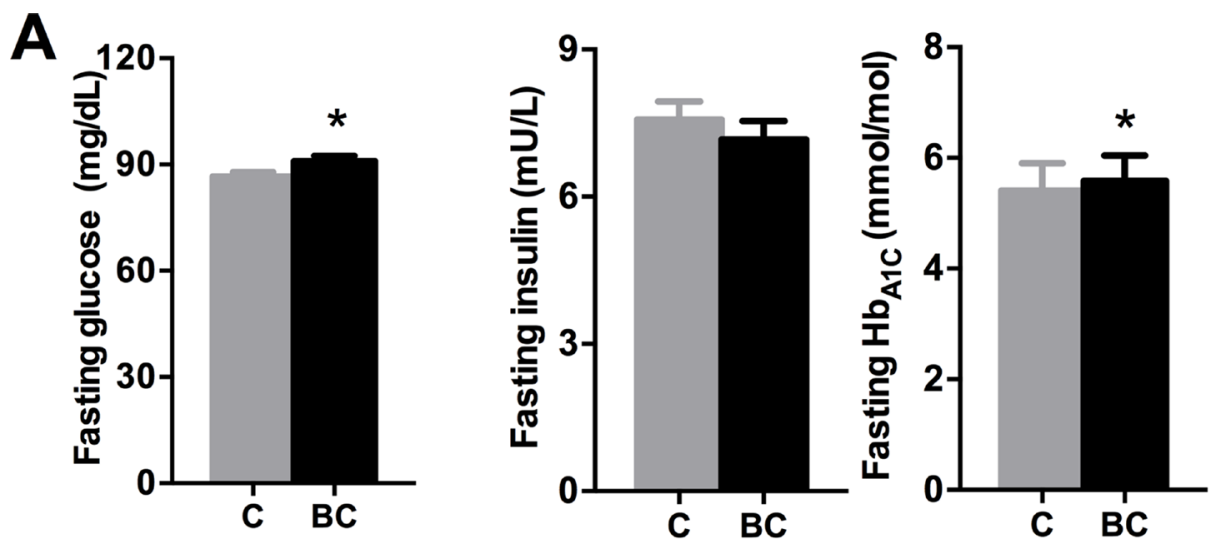

B
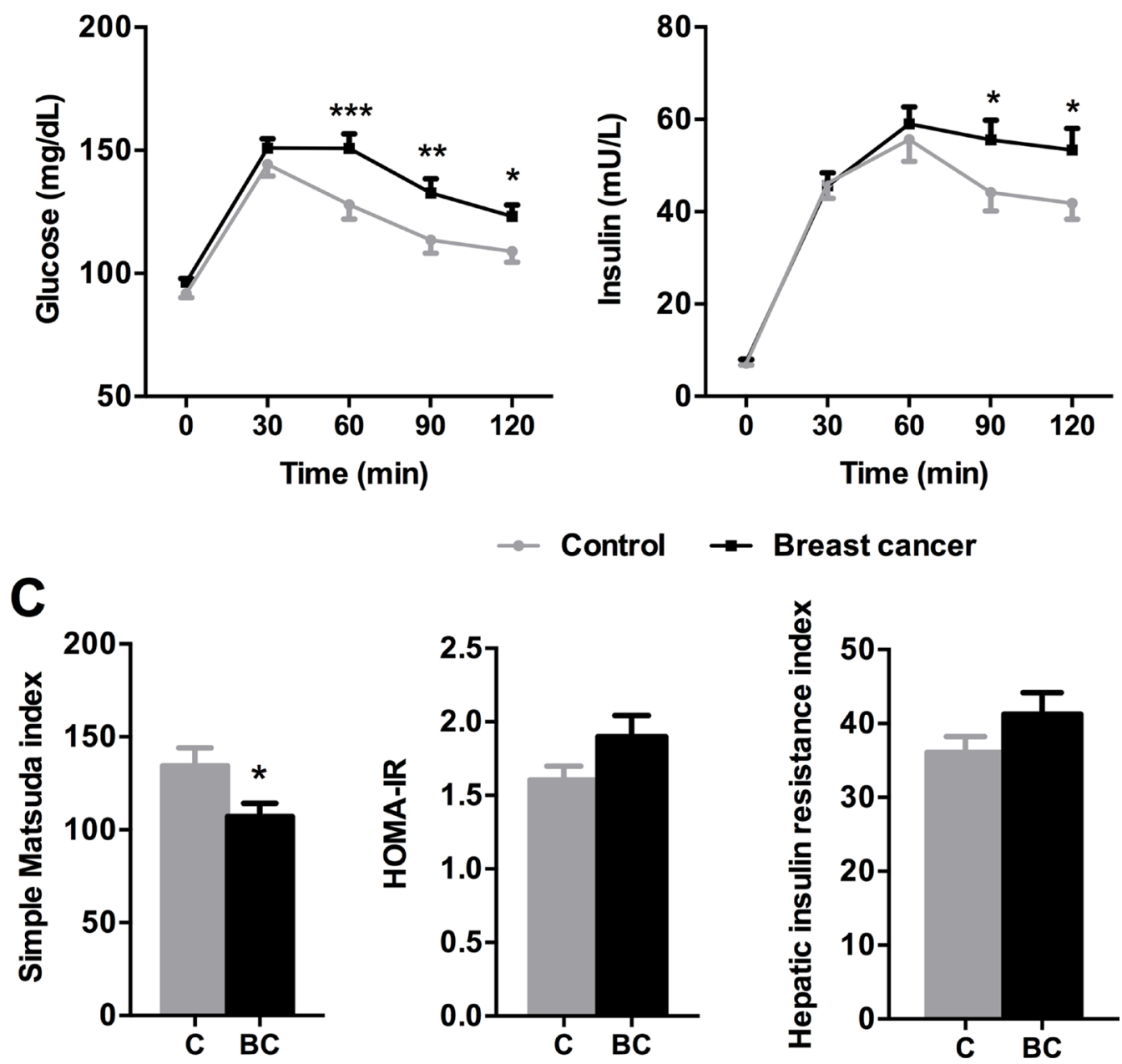

\section{Breast cancer}

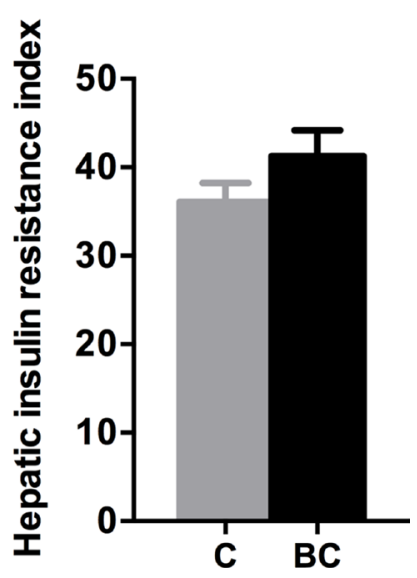

Figure 1: Glucose/insulin metabolism parameters in control and breast cancer patients. (A) Fasting glucose, insulin and glycohemoglobin levels were analyzed. Asterisks $\left({ }^{*} p<0.05\right)$ indicate differences by Mann-Whitney U/Student $t$-test. (B) Glucose and insulin levels during the OGTT. Asterisks $\left({ }^{*} p<0.05 ; * *<0.01 ; * * * p<0.001\right)$ indicate significant differences between groups by Fisher's LSD test. (C) Simple Matsuda index, HOMA-IR and Hepatic insulin resistance index. Asterisks $\left({ }^{*} p<0.05\right)$ indicate differences by Student $t$-test. Values represent means \pm SEM of control $(n=71)$ and breast cancer $(n=77)$ groups. C means control subjects and BC means breast cancer patients. 
postmenopausal subjects. Interestingly, the differences within these groups seemed to be especially drastic in women with BMI > 25 (Supplementary Figure 3C).

\section{The severity of glucose/insulin metabolism} impairment in BCa patients is directly associated to BMI

The analysis implemented when $\mathrm{BMI} \geq 25$ patients were subdivided into overweight $(25 \geq \mathrm{BMI}<30)$ and obese $(\mathrm{BMI} \geq 30)$ showed that fasting glucose levels were significantly increased in obese $\mathrm{BCa}$ patients compared to normal weight and overweight $\mathrm{BCa}$ patients, and to obese controls (Figure 4A). Fasting insulin levels were elevated in obese individuals compared to the respective normal weight controls and obese BCa patients (Figure 4A). Interestingly, glycosylated hemoglobin was only elevated in obese controls compared to normal weight and overweight controls (Figure 4A). In addition, glucose levels during the OGTT remained more elevated in $\mathrm{BCa}$ obese patients than in $\mathrm{BCa}$ overweight patients, compared to their corresponding controls (Figure 4B). Moreover, OGTT curves of obese BCa patients were significantly different to those from normal weight and overweight $\mathrm{BCa}$

\section{A Premenopausal Postmenopausal state state}
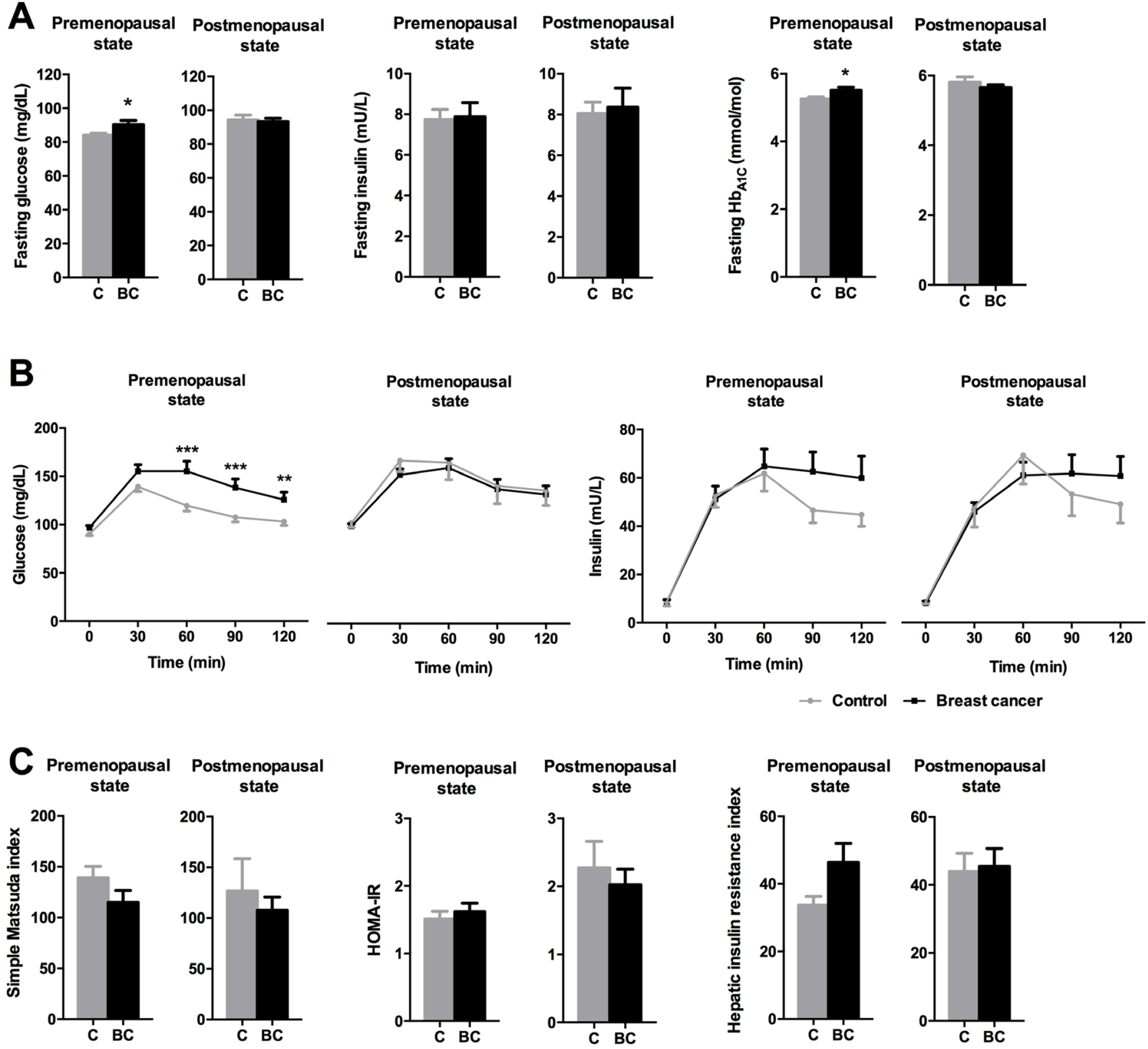

Figure 2: Impact of menopausal status on glucose/insulin metabolism parameters in control and breast cancer patients. (A) Fasting glucose, insulin and glycohemoglobin levels were analyzed. Asterisks $\left({ }^{*} p<0.05\right)$ indicate differences by Student $t$-test. (B) Glucose and insulin levels during the OGTT. Asterisks $(* * p<0.01 ; * * * p<0.001)$ indicate significant differences by Fisher's LSD test. (C) Simple Matsuda index, HOMA-IR and Hepatic insulin resistance index. Values represent means \pm SEM. C means control subjects and $\mathrm{BC}$ means breast cancer patients. 
patients, whereas in controls, OGTT response was only statistically different between normal weight and obese individuals (Supplementary Figure 4). Consistently, insulin levels during the OGTT remained elevated only in $\mathrm{BCa}$ obese patients compared to controls (BCa presence effect: $p=0.012$ ) (Figure 4B). Finally, IR indexes were clearly different between normal weight and obese individuals, but did not reflect an influence of BCa presence (Figure 4C).

\section{Determinants of the association between $\mathrm{BCa}$ and impaired glucose/insulin metabolism}

To ascertain the putative causal relationship between presence of $\mathrm{BCa}$ and impaired glucose/insulin metabolism, we have determined the circulating levels of obesity- and BCa-associated factors. As shown in Figure 5A, certain obesity-associated markers such as triglycerides (TG)
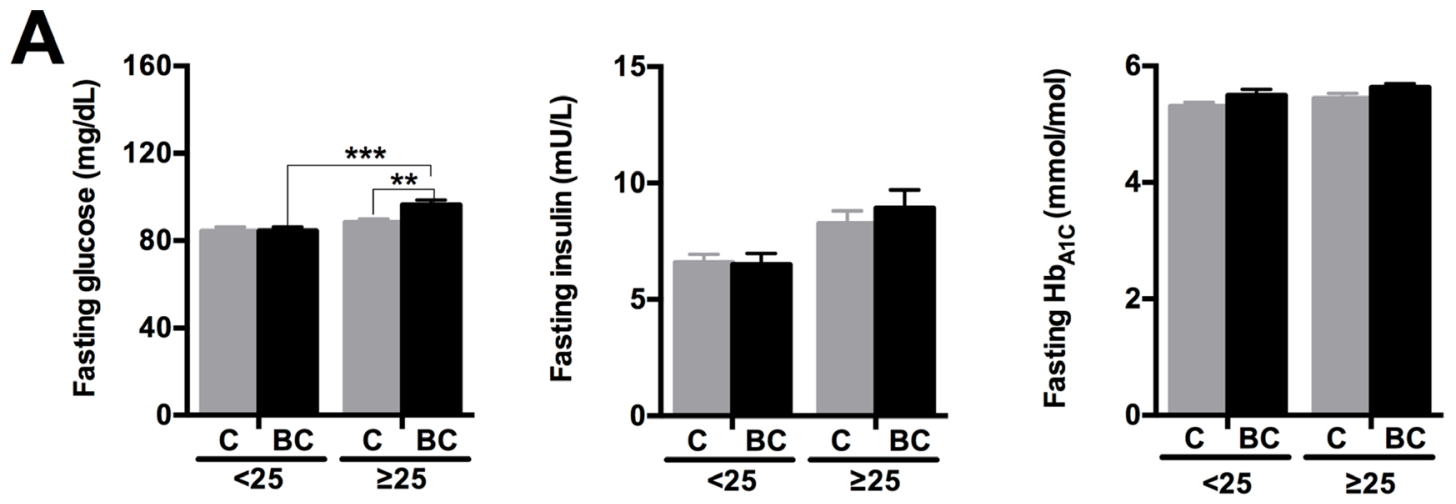

B

BMI $<25$
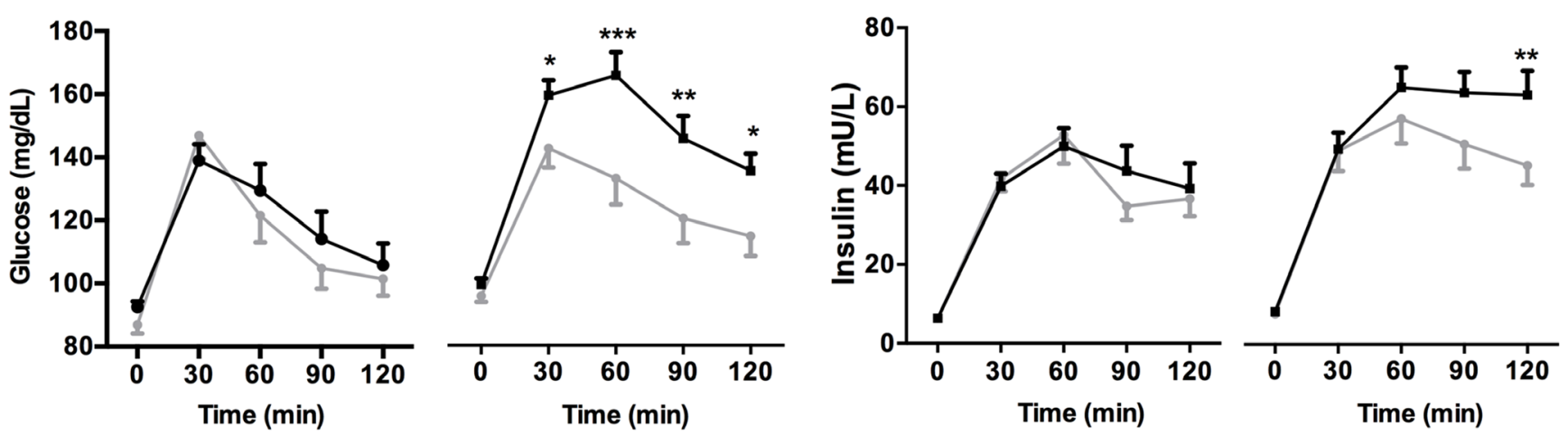

$\rightarrow$ Control

Breast cancer
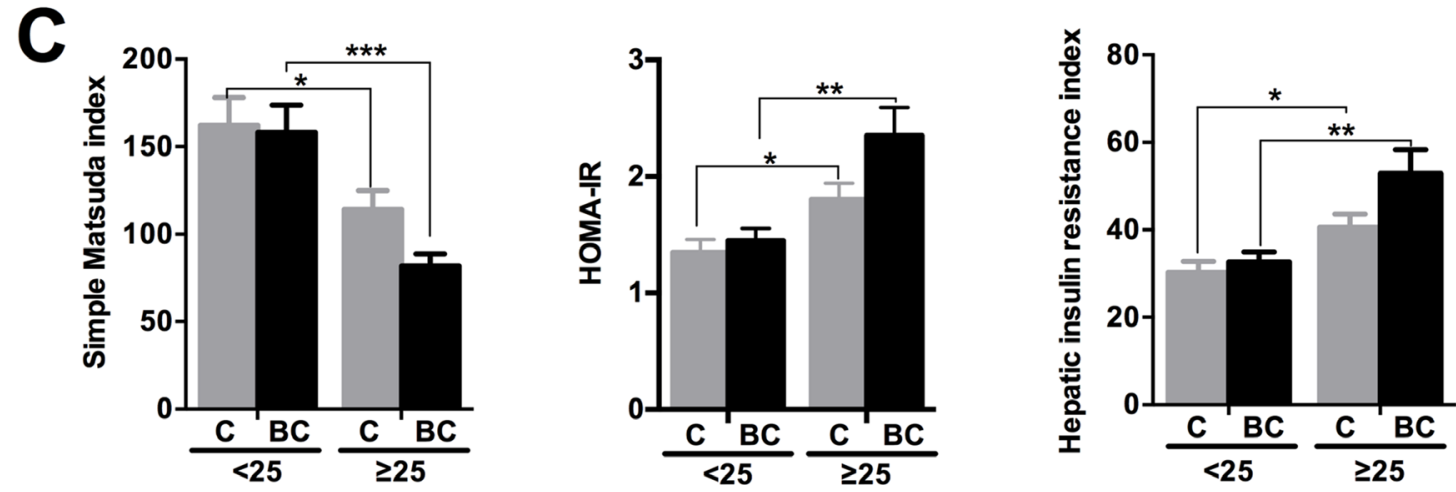

Figure 3: Glucose/insulin metabolism parameters in normal weight $(\mathrm{BMI}<25)$ and overweight/obese $(\mathrm{BMI} \geq 25)$ control and breast cancer patients. (A) Fasting glucose, insulin and glycohemoglobin levels. (B) Glucose and insulin levels during the OGTT. (C) Simple Matsuda index, HOMA-IR and Hepatic insulin resistance index. Values represent mean \pm SEM of each experimental group (normal weight $n=60$; overweight $n=88$ ). Asterisks $(* p<0.05 ; * *<0.01$; $* * *<0.001)$ indicate significant differences by Bonferroni/Mann-Whitney U/Fisher's LSD post-hoc tests. C means control subjects and BC means breast cancer patients. 


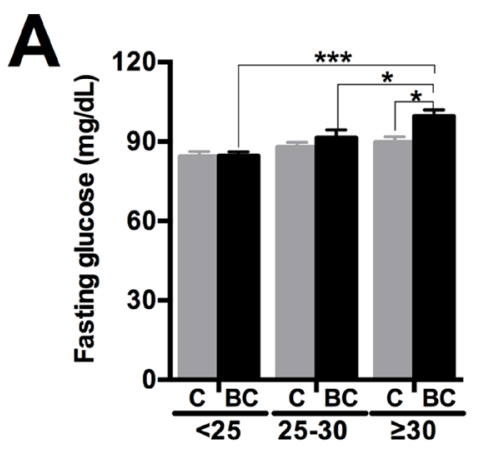

B

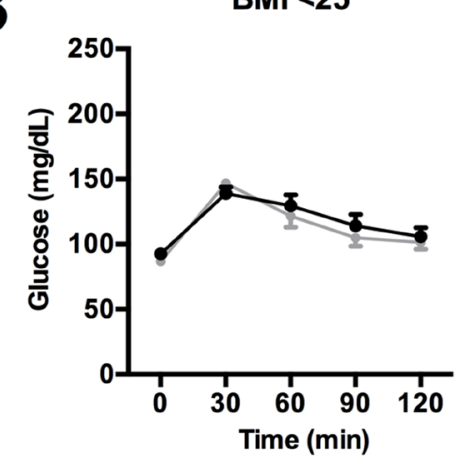

BMI $<25$

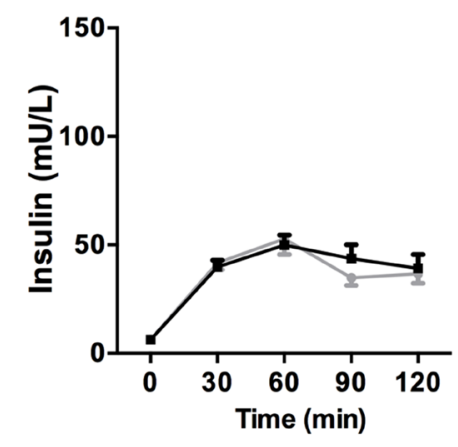

C

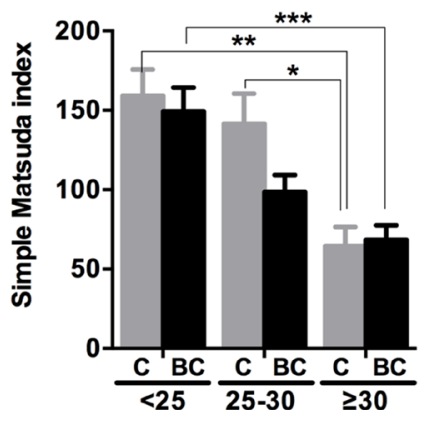

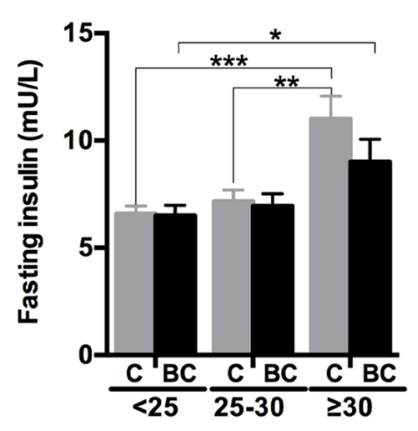

BMI 25-30
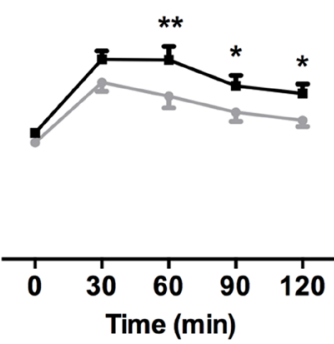

BMI 25-30
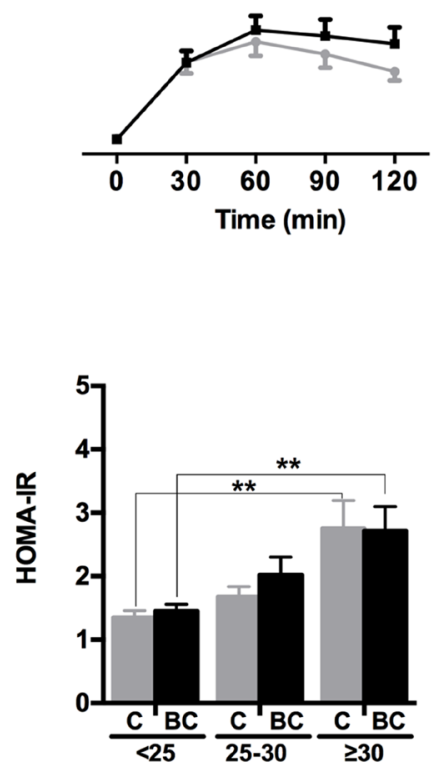

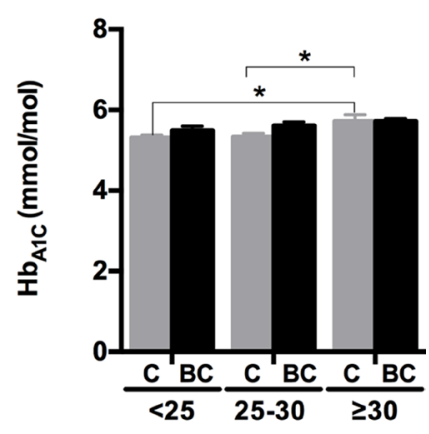

BMI $\geq 30$
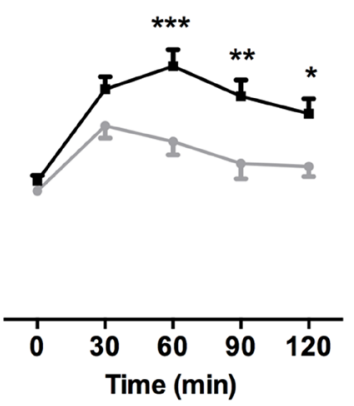

BMI $\geq 30$

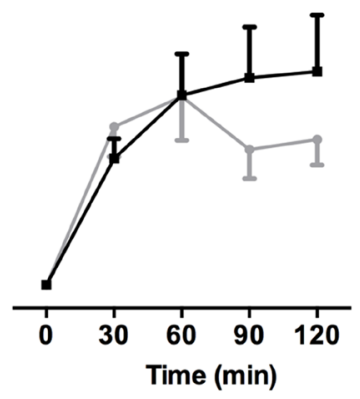

$\rightarrow$ Control $\rightarrow$ Breast cancer

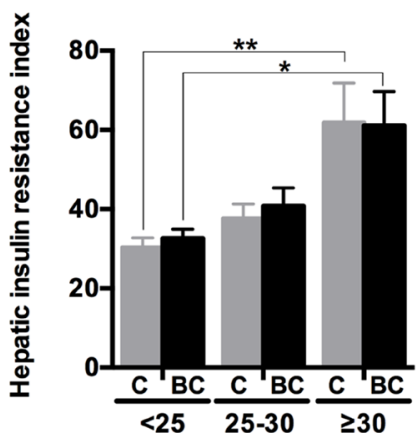

Figure 4: Glucose/insulin metabolism parameters in normal weight (BMI $<25)$, overweight $(\mathrm{BMI} \geq 25<30)$ and obese (BMI $\geq 30$ ) control and breast cancer patients. (A) Fasting glucose, insulin and glycohemoglobin levels. (B) Glucose and insulin levels during the OGTT. (C) Simple Matsuda index, HOMA-IR and Hepatic insulin resistance index. Values represent mean \pm SEM of each experimental group (normal weight $n=60$; overweight $n=54$; obese $n=34$ ). Asterisks $(* p<0.05 ; * * p<0.01 ; * * * p<0.001$ ) indicate significant differences by Bonferroni/Mann-Whitney U/Fisher's LSD post-hoc tests. C means control subjects and BC means breast cancer patients. 
or non-esterified fatty acids (NEFA) were increased in $\mathrm{BCa}$ patients compared to controls, while leptin or adiponectin were not significantly altered. Similarly, Vascular Endothelial Growth Factor (VEGF), a tumorderived factor, tended ( $p=0.061)$ to be increased in $\mathrm{BCa}$ patients; while other putative tumor-derived factors such as RANK-L, Hypoxia-inducible Factor 1 alpha (HIF1 $\alpha$ ) or TNF- $\alpha$ were not significantly altered (Figure 5B). Additionally, other hormones [Growth hormone (GH), IGF-I, estrogens, cortisol) were non-significantly altered, except for prolactin, which tended $(p=0.057)$ to be lower in $\mathrm{BCa}$ patients. Consistently, when the patients were stratified according to the BMI, similar changes/tendencies were found (Supplementary Figures 5 and 6), confirming that TG, NEFA, VEGF and prolactin were the factors altered by the presence of $\mathrm{BCa}$, specially in overweight and/or obese patients.

\section{DISCUSSION}

Although $\mathrm{BCa}$ is strongly influenced by obesity, diabetes and metabolic syndrome [2-4, 6-8, 11], the pathophysiological mechanisms and the molecular and cellular determinants underlying this association are not fully elucidated [26]. Among the players proposed, IR represents a leading candidate, in that it is a major component of metabolic syndrome, diabetes and other metabolic diseases, as well as of several cancer types including $\mathrm{BCa}$. However, the results reported hitherto are controversial [15-19], likely due to the differences in the methods employed to assess IR, and disparities in the intrinsic particularities of the study cohorts, as is the case of BMI or menopausal state. This study was devised to unequivocally unveil the potential association between $\mathrm{BCa}$ and IR, by assessing this parameter more accurately, and by stratifying the patients appropriately by BMI and menopausal state. Thereby, this prospective study revealed the existence of a clear association between BCa and IR, which is particularly drastic in premenopausal women and is strongly influenced by BMI.

Indeed, the results presented herein demonstrate, for first time, that premenopausal overweight and obese $\mathrm{BCa}$ patients exhibit a significantly impaired OGTT compared to that of BMI- and menopausal state-matched controls, whereas this association is not patent in premenopausal normal weight patients nor in postmenopausal women. Interestingly, previous studies have shown that overweight BCa patients near to the initiation of chemotherapy $(n=8)$ have worsened OGTT compared to age- and BMI-matched controls [22], that OGTT improved after 6 months chemotherapy in ten previously undiagnosed diabetic postmenopausal BCa women [27], and that non-diabetic overweight $\mathrm{BCa}$ patients present higher fasting glucose and insulin levels [13]. However, this study provides the first evidence that naive, untreated $\mathrm{BCa}$ patients exhibit worsened OGTT compared to matched controls, and that this is predominantly observed in premenopausal women and drastically influenced by BMI. Indeed, normal weight premenopausal $\mathrm{BCa}$ patients did not exhibit signs of impaired glucose tolerance or IR compared to normal weight controls, thereby suggesting that, under normal weight conditions, presence of $\mathrm{BCa}$ is not associated to worsened glucose/insulin metabolism. Conversely, under conditions of deteriorated glucose management (overweight/obesity), presence of $\mathrm{BCa}$ was associated to aggravated IR and impaired glucose homeostasis, which could even worsen the outcome of overweight/ obese BCa patients. Importantly, our observation could be related to the fact that the relative risk of mortality is higher in obese BCa patients compared to controls [3, 4, $28,29]$. In contrast to premenopausal women and despite obesity and metabolic syndrome are associated to worst $\mathrm{BCa}$ outcome and survival especially in postmenopause [2-4, 11, 28], BCa postmenopausal patients did not present altered glucose/insulin metabolism irrespective of BMI. This observation could be associated to the fact that postmenopausal woman already exhibit impaired glucose/insulin metabolism compared to premenopausal women ([30, 31] and data presented herein). In any case, it should be noted that a limitation of this study is that body fat mass and body fat distribution of the patients, two parameters that could be influencing insulin resistance, were not determined, and, therefore, this issue warrants future investigation

Nevertheless, it is yet uncertain whether dysregulated glucose/insulin homeostasis, particularly in obese women, could cause/enhance $\mathrm{BCa}$ development and progression and/or, on the other hand, $\mathrm{BCa}$ presence could induce/exacerbate a situation of impaired glucose/ insulin metabolism. Likewise, the precise mechanisms and cellular/molecular players underlying this uni- or bidirectional relationship are not fully known. Several studies have provided evidence favoring the notion that a reciprocal influence can occur, although the weight of the data in each direction is not balanced. Specifically, mounting evidence indicate that several obesity-associated factors such as insulin, leptin, adiponectin or triglycerides can contribute to the development and/or progression of several cancer types, including $\mathrm{BCa}[32,33]$. Likewise, there is also data supporting the contention that the tumor could influence whole body glucose/insulin metabolism through the secretion of certain factors [34-40]. In an attempt to shed some light in this issue, we determined circulating concentrations of selected obesity- and tumorderived factors. Consistent with previous studies [20], glucose and glycosylated hemoglobin were elevated in $\mathrm{BCa}$ patients, especially in those exhibiting a more pronounced impairment of glucose/insulin metabolism (premenopausal patients with higher BMI) compared to controls. However, insulin levels were not significantly altered in the experimental groups, an observation that compares well with previous studies [21], and suggests 
that insulin is unlikely to be solely responsible for this association. Interestingly, TG and NEFA, other IRassociated markers $[41,42]$, which are involved in $\mathrm{BCa}$ development/progression [43, 44], were elevated in $\mathrm{BCa}$ patients compared to BMI-matched controls, thus underscoring the interest in further studying the potential contribution and underlying mechanisms of these factors into BCa. Additionally, from the tumor-associated factors analyzed (VEGF, RANK-L, TNF-alpha and HIF-1alpha) [45-48] that could be putatively associated to the impaired
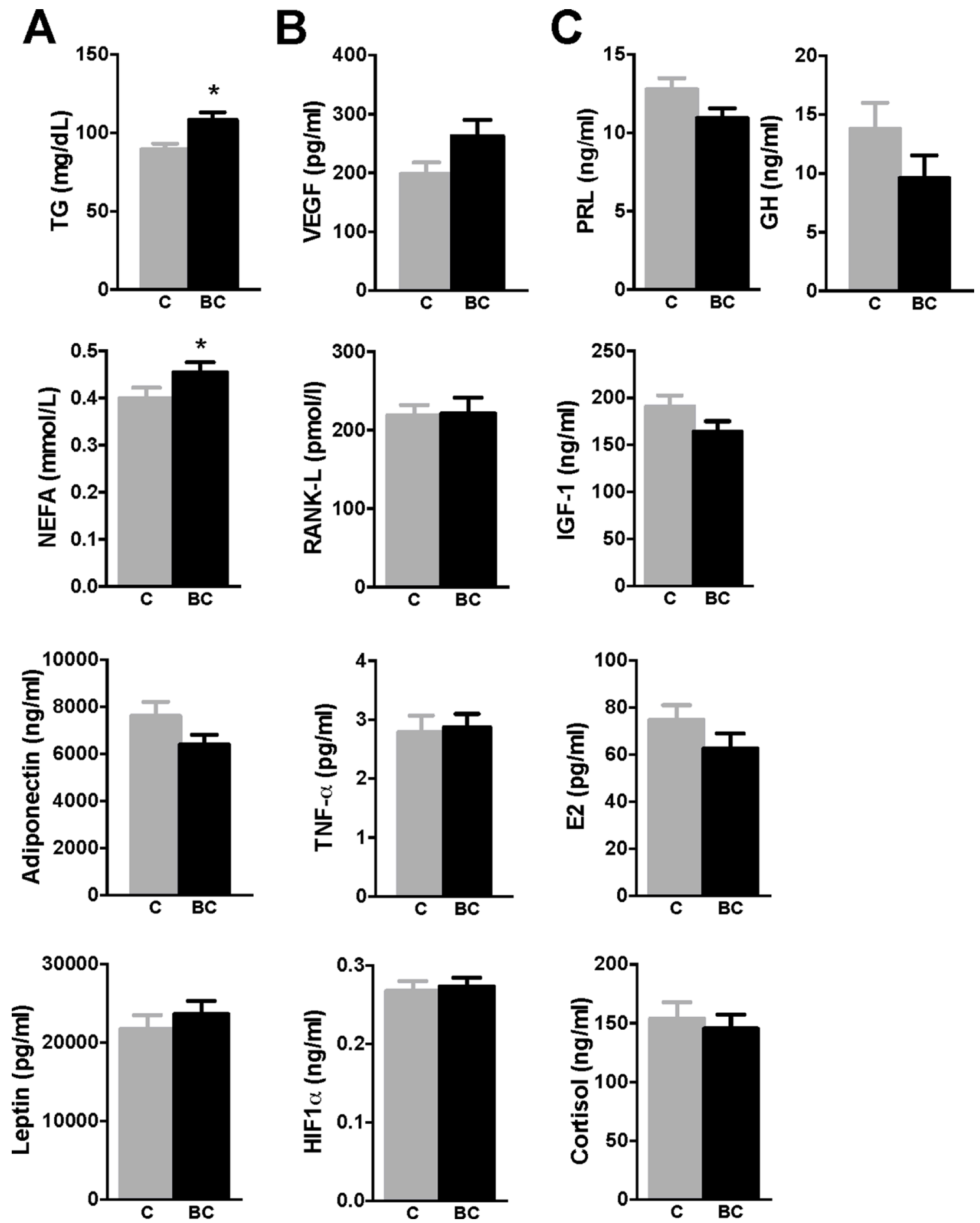

Figure 5: Circulating levels of relevant hormones, factors and molecules in control and breast cancer patients. (A) Obesity-associated markers (TG, NEFA, adiponectin, leptin), (B) tumor-derived factors (VEGF, RANK-L, TNF- $\alpha$, HIF-1 $\alpha$ ) and (C) other relevant hormones [PRL, IGF-I, estrogens (E2), cortisol, GH] were determined in plasma of cohort individuals by specific ELISAs. Values represent mean \pm SEM of each experimental group (control $n=71$; breast cancer $n=77$ ). Asterisks $(* p<0.05)$ indicate significant differences between groups by Student $t$-test/Mann-Whitney $U$ test. C means control subjects and BC means breast cancer patients. 
glucose/insulin metabolism [35, 39, 40, 49], only VEGF was found overtly elevated in $\mathrm{BCa}$ patients compared to controls. The bidirectional relationship between VEGF and impaired glucose/insulin homeostasis in $\mathrm{BCa}$ has not been studied in detail hitherto, apart from some insightful animal studies indicating that VEGF-C overexpression induces weight gain and IR [34]. Our findings suggest that this putative interaction deserves a more careful examination, as the data presented herein provide evidence that a multifactorial causative reciprocal interconnection may underlie the pathological association between IR and $\mathrm{BCa}$ in overweight/obese premenopausal women, wherein insulin-resistance associated factors (glucose, TG, FFA) could potentiate $\mathrm{BCa}$ development/progression while tumor-derived factors (VEGF) could aggravate IR.

In summary, our results indicate that $\mathrm{BCa}$ presence is associated to higher IR in overweight/obese premenopausal woman but not in premenopausal normal weight patients or in postmenopausal woman. Further, our findings suggest a bidirectional relationship between dysregulated glucose/insulin metabolism and $\mathrm{BCa}$, as both, tumor- and IR-related markers are correlated with the severity of glucose/insulin metabolism impairment in overweight/obese premenopausal BCa patients.

\section{MATERIALS AND METHODS}

\section{Ethics, consent and permissions}

The study protocol was approved by the Human Investigation Review Committee of the Reina Sofia University Hospital (HURS, Cordoba, Spain). Patient management followed the guidelines established by the internal "Breast Cancer Patients Protocol". We had all necessary consents from patients involved in the study, including consent to participate in the study.

\section{Participants}

Between 2011 and 2015, women received in the Mammary Gland Unit at the HURS with suspect of nonfamilial, sporadic $\mathrm{BCa}$, considered within a group of common risk, image-diagnosed within BIRADS (Breast Imaging Reporting and Data System) grade 4-5 and subjected to core-needle biopsy of the mammary gland were invited to participate in this study. Particularly, 148 women (mean age: $49.3 \pm 0.9$; range: $21-81$ ) accepted to participate in this prospective study. After the diagnosis (core-needle biopsy), patients were classified as "Control" $(n=77)$ or "Breast Cancer" $(n=71)$.

\section{Study design}

The clinical protocol included: 1) collection of personal data, family history, associated pathologies and menopausal state; 2) evaluation of body mass index, waist perimeter and blood pressure; 3) collection of blood in fasting state; and 4) OGTT performance. According to current laws and legal procedures, all the personal information collected was confidential, and all biological samples and the results obtained were codified. Those patients with family history of $\mathrm{BCa}$ or diseases related to glucose metabolism (diabetes) and other chronic illnesses were excluded.

\section{Blood sampling, OGTT and biochemical analysis}

Sessions started between 8:30-9:30 am and all participants were fasted for 8-12 hours (no food or drink except for water). Fasting blood samples were obtained for biochemical analysis. Then, two fasting samples were drawn (times $-15 \mathrm{~min}$ and $0 \mathrm{~min}$, respectively); immediately after, an oral overload of 75 gr of glucose drink was provided. At 30, 60, 90 and $120 \mathrm{~min}$ [50], blood samples were obtained and spread into three tubes (BD Vacutainer, New Jersey, US): EDTAK - treated plasma, oxalate-treated plasma and serum-separating tubes. Plasma and oxalate-treated blood were obtained after centrifugation of $10 \mathrm{~min}$ at $3000 \mathrm{rpm}\left(4^{\circ} \mathrm{C}\right)$, while serum was obtained by centrifugation of $20 \mathrm{~min}$ at 2500 rpm (room temperature). Plasma was frozen at $-80^{\circ} \mathrm{C}$, while $500 \mu \mathrm{l}$ of each oxalate-plasma and serum sample were used for glucose (Hexokinase/G-6-PDH technique) and insulin (ARCHITEC i2000SR, Abbott Diagnostic) determinations.

\section{Determination of insulin sensitivity/resistance indexes}

Fasting indexes were calculated using the formula: HOMA-IR $=$ [fasting insulin $(\mathrm{mU} / \mathrm{L}) *$ fasting glucose $(\mathrm{mmol} / \mathrm{L})] / 22.5$ and Hepatic insulin resistance Index $($ HIRI $)=$ fasting glucose*fasting insulin. From OGTT, Simple Matsuda Index (an index to evaluate whole body insulin sensitivity) was calculated as: $\mathrm{SMI}=10.000$ /square root of [fasting glucose*fasting insulin]*[glucose at time $120 *$ insulin at time 120].

\section{Measurements of circulating hormones and metabolites}

Fasting blood was used to determine glucose, insulin, glycohemoglobin, lipids (non-esterified fatty acids [NEFA (Wako Chemicals GmbH, Neuss, DE)], total cholesterol [TC], low-density lipoprotein cholesterol [LDL], high density lipoprotein cholesterol [HDL] and triglycerides [TG]). Also, circulating levels of GH, IGF-1, cortisol and PRL (DRG International, Inc., US); leptin, TNF- $\alpha$, VEGF (Invitrogen, CA, US), adiponectin (R\&D Systems, MN, US), RANK-L (BioVendor, CR, US), HIF1 $\alpha$ (RayBiotech, GA, US) and estradiol (Life Technologies, CA, US) were measured using commercial 
ELISA kits. All the information regarding each assay can be accessed at the company website.

\section{Statistics}

To evaluate data variation in OGTT measurements, analysis of variance for repeated measures (2-way ANOVA) was performed, followed by Fisher's correction test for multiple comparisons (the effect of $\mathrm{BCa} /$ menopause/BMI and time was determined). To analyze the influence of age in the results derived from the OGTT, a linear regression analysis was carried out. Moreover, Student $t$-test, Mann-Whitney and 2-way ANOVA (followed by Bonferroni's post-hoc test) tests were used, according to the number of experimental groups analyzed and the normality of the data. All data are expressed as mean \pm SEM. Differences were considered to be significant when $p<0.05$. GraphPad Prism 6.0 software (La Jolla, CA) and SPSS 24.0 (IBM) were used for the statistical analysis.

\section{Author contributions}

RML, LMLS, AVO and MDG performed all the experiments and analyses. IML, MCG and ALSR coordinated patient recruitment and obtainment of samples. EMYS, MAB and JLM provided valuable feedback during the project and revised the manuscript draft. RML, MDG and JPC conceived the study, participated in its design and coordination and drafted the manuscript. All authors contributed to the interpretation and discussion of the findings and approved the final manuscript.

\section{ACKNOWLEDGMENTS}

We thank all the individuals who took part in these studies and all the researchers, clinicians, technicians, and administrative staff who enabled this work to be carried out.

\section{CONFLICTS OF INTEREST}

The authors declare that they have no conflicts of interest.

\section{FUNDING}

This work has been supported by MINECO [BFU2013-43282-R and BFU2016-80360-R to Justo P. Castaño, Juan de la Cierva grant (JCI-2009-05675) to Laura M Lopez-Sanchez], Ministerio de Educación, Cultura y Deporte (FPU12/01086 to Alicia Villa-OSaba), Instituto de Salud Carlos III, co-funded by European Union (ERDF/ESF, "Investing in your future") [PI1300651 and PI16/00264 to Raúl M. Luque, Miguel Servet grant (CP15/00156) to Manuel D. Gahete], Junta de Andalucía (BIO-0139, CTS-1406, PI-0639-2012, PI-05412013 to Raúl M. Luque, Justo P. Castaño and Manuel D. Gahete), and CIBERobn. CIBER is an initiative of Instituto de Salud Carlos III, Ministerio de Sanidad, Servicios Sociales e Igualdad, Spain.

\section{REFERENCES}

1. Siegel RL, Miller KD, Jemal A. Cancer Statistics, 2017. CA Cancer J Clin. 2017; 67:7-30.

2. Simone V, D’Avenia M, Argentiero A, Felici C, Rizzo FM, De Pergola G, Silvestris F. Obesity and breast cancer: molecular interconnections and potential clinical applications. Oncologist. 2016; 21:404-17.

3. Jiralespong S, Goodwin P. Obesity and breast cancer prognosis: evidence, challenges, and opportunities. J Clin Oncol. 2016; 34:4203-16.

4. Goodwin PJ. Obesity, insulin resistance and breast cancer outcomes. Breast. 2015; 24:S56-S9.

5. Donohoe CL, Lysaght J, O'Sullivan J, Reynolds JV. Emerging concepts linking obesity with the hallmarks of cancer. Trends Endocrinol Metab. 2017; 28:46-62.

6. Xue F, Michels KB. Diabetes, metabolic syndrome, and breast cancer: a review of the current evidence. Am J Clin Nutr. 2007; 86:s823-35.

7. Berrino F, Villarini A, Traina A, Bonanni B, Panico S, Mano MP, Mercandino A, Galasso R, Barbero M, Simeoni M, Bassi MC, Consolaro E, Johansson H, et al. Metabolic syndrome and breast cancer prognosis. Breast Cancer Res Treat. 2014; 147:159-65.

8. Agnoli C, Grioni S, Sieri S, Sacerdote C, Ricceri F, Tumino R, Frasca G, Pala V, Mattiello A, Chiodini P, Iacoviello L, De Curtis A, Panico S, et al. Metabolic syndrome and breast cancer risk: a case-cohort study nested in a multicentre italian cohort. PLoS ONE. 2015; 10:e0128891.

9. Arcidiacono B, Iiritano S, Nocera A, Possidente $\mathrm{K}$, Nevolo MT, Ventura V, Foti D, Chiefari E, Brunetti A. Insulin resistance and cancer risk: an overview of the pathogenetic mechanisms. Exp Diabetes Res. 2012; 2012:789174.

10. Nam S, Park S, Park HS, Kim S, Kim JY, Kim SI. Association between insulin resistance and luminal B subtype breast cancer in postmenopausal women. Medicine. 2016; 95:e2825.

11. Rose DP, Gracheck PJ, Vona-Davis L. The interactions of obesity, inflammation and insulin resistance in breast cancer. Cancers. 2015; 7:2147-68.

12. Rose DP, Haffner SM, Baillargeon J. Adiposity, the metabolic syndrome, and breast cancer in African-American and white American women. Endocr Rev. 2007; 28:763-77.

13. Ferroni P, Riondino S, Laudisi A, Portarena I, Formica V, Alessandroni J, D’Alessandro R, Orlandi A, Costarelli L, Cavaliere F, Guadagni F, Roselli M. Pretreatment insulin levels as a prognostic factor for breast cancer progression. Oncologist. 2016; 21:1041-9. 
14. Protani M, Coory M, Martin JH. Effect of obesity on survival of women with breast cancer: systematic review and metaanalysis. Breast Cancer Res Treat. 2010; 123:627-35.

15. Autier P, Koechlin A, Boniol M, Mullie P, Bolli G, Rosenstock J, Boyle P. Serum insulin and C-peptide concentration and breast cancer: a meta-analysis. Cancer Causes Control. 2013; 24:873-83.

16. Yam D, Fink A, Mashiah A, Ben-Hur E. Hyperinsulinemia in colon, stomach and breast cancer patients. Cancer Lett. 1996; 104:129-32.

17. Eliassen AH, Tworoger SS, Mantzoros CS, Pollak MN, Hankinson SE. Circulating insulin and c-peptide levels and risk of breast cancer among predominately premenopausal women. Cancer Epidemiol Biomarkers Prev. 2007; 16:161-4.

18. Fair AM, Dai Q, Shu XO, Matthews CE, Yu H, Jin F, Gao YT, Zheng W. Energy balance, insulin resistance biomarkers, and breast cancer risk. Cancer Detect Prev. 2007; 31:214-9.

19. Garmendia ML, Pereira A, Alvarado ME, Atalah E. Relation between insulin resistance and breast cancer among Chilean women. Ann Epidemiol. 2007; 17:403-9.

20. Muti P, Quattrin T, Grant BJ, Krogh V, Micheli A, Schunemann HJ, Ram M, Freudenheim JL, Sieri S, Trevisan M, Berrino F. Fasting glucose is a risk factor for breast cancer: a prospective study. Cancer Epidemiol Biomarkers Prev. 2002; 11:1361-8.

21. Hernandez AV, Guarnizo M, Miranda Y, Pasupuleti V, Deshpande A, Paico S, Lenti H, Ganoza S, Montalvo L, Thota P, Lazaro H. Association between insulin resistance and breast carcinoma: a systematic review and metaanalysis. PLoS One. 2014; 9:e99317.

22. Bell KE, Di Sebastiano KM, Vance V, Hanning R, Mitchell A, Quadrilatero J, Russell C, Dubin JA, Bahl M, Califaretti N, Campbell C, Mourtzakis M. A comprehensive metabolic evaluation reveals impaired glucose metabolism and dyslipidemia in breast cancer patients early in the disease trajectory. Clin Nutr. 2014; 33:550-7.

23. Barrett-Connor E. Postmenopausal estrogen replacement and breast cancer. N Engl J Med. 1989; 321:319-20.

24. Anderson KN, Schwab RB, Martinez ME. Reproductive risk factors and breast cancer subtypes: a review of the literature. Breast Cancer Res Treat. 2014; 144:1-10.

25. Jain R, Strickler HD, Fine E, Sparano JA. Clinical studies examining the impact of obesity on breast cancer risk and prognosis. J Mammary Gland Biol Neoplasia. 2013; 18:257-66.

26. Klil-Drori AJ, Azoulay L, Pollak MN. Cancer, obesity, diabetes, and antidiabetic drugs: is the fog clearing? Nat Rev Clin Oncol. 2017; 14:85-99.

27. Chala E, Manes C, Iliades H, Skaragkas G, Mouratidou D, Kapantais E. Insulin resistance, growth factors and cytokine levels in overweight women with breast cancer before and after chemotherapy. Hormones. 2006; 5:137-46.
28. Protani M, Coory M, Martin JH. Effect of obesity on survival of women with breast cancer: systematic review and metaanalysis. Breast Cancer Res Treat. 2010; 123:627-35.

29. Chan DS, Vieira AR, Aune D, Bandera EV, Greenwood DC, McTiernan A, Navarro Rosenblatt D, Thune I, Vieira R, Norat T. Body mass index and survival in women with breast cancer-systematic literature review and meta-analysis of 82 follow-up studies. Ann Oncol. 2014; 25:1901-14.

30. Carr MC. The emergence of the metabolic syndrome with menopause. J Clin Endocrinol Metab. 2003; 88:2404-11.

31. Spencer CP, Godsland IF, Stevenson JC. Is there a menopausal metabolic syndrome? Gynecol Endocrinol. 1997; 11:341-55.

32. Saxena NK, Sharma D. Multifaceted leptin network: the molecular connection between obesity and breast cancer. J Mammary Gland Biol Neoplasia. 2013; 18:309-20.

33. Belardi V, Gallagher EJ, Novosyadlyy R, LeRoith D. Insulin and IGFs in obesity-related breast cancer. J Mammary Gland Biol Neoplasia. 2013; 18:277-89.

34. Karaman S, Hollmén M, Yoon SY, Alkan HF, Alitalo K, Wolfrum C, Detmar M. Transgenic overexpression of VEGF-C induces weight gain and insulin resistance in mice. Sci Rep. 2016; 6:31566.

35. Jiang C, Qu A, Matsubara T, Chanturiya T, Jou W, Gavrilova O, Shah YM, Gonzalez FJ. Disruption of hypoxia-inducible factor 1 in adipocytes improves insulin sensitivity and decreases adiposity in high-fat diet-fed mice. Diabetes. 2011; 60:2484-95.

36. Ando Y, Shinozawa Y, Iijima Y, Yu BC, Sone M, Ooi Y, Watanaka Y, Chida K, Hakuno F, Takahashi SI. Tumor necrosis factor (TNF)- $\alpha$-induced repression of GKAP42 protein levels through cGMP-dependent kinase (cGK)I $\alpha$ causes insulin resistance in 3T3-L1 adipocytes. J Biol Chem. 2015; 290:5881-92.

37. Halberg N, Khan T, Trujillo ME, Wernstedt-Asterholm I, Attie AD, Sherwani S, Wang ZV, Landskroner-Eiger S, Dineen S, Magalang UJ, Brekken RA, Scherer PE. Hypoxia-inducible factor $1 \alpha$ induces fibrosis and insulin resistance in white adipose tissue. Mol Cell Biol. 2009; 29:4467-83.

38. Plomgaard P, Bouzakri K, Krogh-Madsen R, Mittendorfer B, Zierath JR, Pedersen BK. Tumor necrosis factor- $\alpha$ induces skeletal muscle insulin resistance in healthy human subjects via inhibition of Akt substrate 160 phosphorylation. Diabetes. 2005; 54:2939-45.

39. Honek J, Seki T, Iwamoto H, Fischer C, Li J, Lim S, Samani NJ, Zang J, Cao Y. Modulation of age-related insulin sensitivity by VEGF-dependent vascular plasticity in adipose tissues. Proc Natl Acad Sci USA. 2014; 111:14906-11.

40. Kiechl S, Wittmann J, Giaccari A, Knoflach M, Willeit P, Bozec A, Moschen AR, Muscogiuri G, Sorice GP, Kireva T, Summerer M, Wirtz S, Luther J, et al. Blockade of receptor activator of nuclear factor-kappaB (RANKL) signaling 
improves hepatic insulin resistance and prevents development of diabetes mellitus. Nat Med. 2013; 19:358-63.

41. Jung UJ, Choi MS. Obesity and its metabolic complications: the role of adipokines and the relationship between obesity, inflammation, insulin resistance, dyslipidemia and nonalcoholic fatty liver disease. Int J Mol Sci. 2014; 15:6184-223.

42. Li N, Fu J, Koonen DP, Kuivenhoven JA, Snieder H, Hofker MH. Are hypertriglyceridemia and low HDL causal factors in the development of insulin resistance? Atherosclerosis. 2014; 233:130-8.

43. MacLennan M, Ma DW. Role of dietary fatty acids in mammary gland development and breast cancer. Breast Cancer Res. 2010; 12:211.

44. Ulmer H, Borena W, Rapp K, Klenk J, Strasak A, Diem G, Concin H, Nagel G. Serum triglyceride concentrations and cancer risk in a large cohort study in Austria. Br J Cancer. 2009; 101:1202-6.

45. Orosz P, Echtenacher B, Falk W, Ruschoff J, Weber D, Mannel DN. Enhancement of experimental metastasis by tumor necrosis factor. J Exp Med. 1993; 177:1391-8.

46. Costa A, Coradini D, Carrassi A, Erdas R, Sardella A, Daidone MG. Re: Levels of hypoxia-inducible factor1alpha during breast carcinogenesis. J Natl Cancer Inst. 2001; 93:1175-7.
47. Yoneda T, Tanaka S, Hata K. Role of RANKL/RANK in primary and secondary breast cancer. World J Orthop. 2013; 4:178-85.

48. Adams J, Carder PJ, Downey S, Forbes MA, MacLennan K, Allgar V, Kaufman S, Hallam S, Bicknell R, Walker JJ, Cairnduff F, Selby PJ, Perren TJ, et al. Vascular endothelial growth factor (VEGF) in breast cancer: comparison of plasma, serum, and tissue VEGF and microvessel density and effects of tamoxifen. Cancer Res. 2000; 60:2898-905.

49. Ando Y, Shinozawa Y, Iijima Y, Yu BC, Sone M, Ooi Y, Watanaka Y, Chida K, Hakuno F, Takahashi S. Tumor necrosis factor (TNF)-alpha-induced repression of GKAP42 protein levels through cGMP-dependent kinase (cGK)Ialpha causes insulin resistance in 3T3-L1 adipocytes. J Biol Chem. 2015; 290:5881-92.

50. Matsuda M, DeFronzo RA. Insulin sensitivity indices obtained from oral glucose tolerance testing: comparison with the euglycemic insulin clamp. Diabetes Care. 1999; 22:1462-70. 